\title{
Spatial tumor-immune modeling
}

\author{
L. G. DE PILLIS $\dagger^{*}$, D. G. MALLET $\$ \S$ and A. E. RADUNSKAYA $\Uparrow \#$ \\ $\dagger$ Department of Mathematics, Harvey Mudd College, Claremont, CA 91711, USA \\ $\$$ School of Mathematical Sciences, Queensland University of Technology, Brisbane, Australia \\ I| Department of Mathematics, Pomona College, Claremont, CA 91711, USA \\ (Received 28 June 2006; in final form 14 July 2006)
}

\begin{abstract}
In this paper, we carry out an examination of four mechanisms that can potentially lead to changing morphologies in a growing tumor: variations in nutrient consumption rates, cellular adhesion, excessive consumption of nutrients by tumor cells and immune cell interactions with the tumor. We present numerical simulations using a hybrid PDE-cellular automata (CA) model demonstrating the effects of each mechanism before discussing hypotheses about the contribution of each mechanism to morphology change.
\end{abstract}

Keywords: Tumor; Immune; Cellular automata; Cellular adhesion; Tumor morphology

\section{Introduction}

Over the past three to four decades, tumor growth and the dynamics of the immune system have been a significant focus for both experimentalists and mathematical modelers. Mathematical modeling in this area stems from the early chemical diffusion and differential equation models of Burton [11] and Greenspan [31] and has grown into an extensive field of literature with studies presented using partial and ordinary differential equations (PDEs and ODEs) (see for example [12,13,41,46,54]), cellular automata (CA) (see for example $[2,7,26,36,43,49])$ and many statistically based studies. An excellent review of mathematical models of tumor growth and some of the closely related literature was recently given by Araujo and McElwain [5].

The mathematical modeling of the immune system in its various roles is also a rich field of study. For example, Perelson provides a substantial review of the immune system before demonstrating the process of modeling the immune system from the point of view of a physicist [52]. Ordinary differential equations are widely used to model the kinetics of the immune response as illustrated in the work of Merrill [45], Callewaert et al. [14], and Perelson and Macken [51]. CA methods have also arisen in immune system modeling such as in the "IMMSIM" model of Seiden and Celada [59].

Bellomo and Maini [8] note that a significant issue in the modeling of biological systems is the multiscale nature of many such systems. They discuss three distinct but linked levels: the sub-cellular level which covers gene expression, receptor binding and cellular signaling; the

\footnotetext{
*Corresponding author. Email: depillis@hmc.edu

§ Email: dg.mallet@qut.edu.au

\# Email: aradunskaya@pomona.edu
}

Computational and Mathematical Methods in Medicine 
cellular level where, for example, cellular interactions regulated by sub-cellular signals are considered; and the macroscopic level. The cellular level of biological systems is often modeled using nonlinear integro-differential equations or by discrete, agent-based models $[50,53,57]$. In relation to the cellular level of tumor growth, when cell proliferation is not suppressed by the immune system, tumor cells may condense to form a solid mass, leading researchers to develop an interest in descriptions of tumor growth at the macroscopic scale, [9]. At this macroscopic level we can study interactions between the host and macroscopic boundaries, such as tumor boundaries, or the behavior of aggregate cell populations which might lead to tissue heterogeneity. Such systems can be investigated using moving boundary, nonlinear partial differential equation systems, via level-set methods, or using mean-field models, [16,17,19]. Greller et al. [32] provide a generalized conceptual foundation for the modeling of tumor growth and heterogeneity produced therein.

The model developed here couples the growth of a tumor mass with the response of the immune system. We use partial differential equations to provide a macroscopic description of relevant nutrients, combined with a discrete strategy which considers the behavior of individual cells and their interactions, at the cellular level. Thus, the model can be considered a hybrid of the macroscopic approach and an agent-based approach. Greller et al. [32] offer astute reasons for taking care when taking the continuum limit of a microscopic process. In this case, the validity of a continuum model of the molecular concentrations has been justified historically by the use of the continuous diffusion equation to model the diffusion of small molecules through tissue, e.g. [23,24,65]. Furthermore, the timescale of molecular diffusion is short relative to that of tumor cell growth, justifying the modeling of the small molecule population as an aggregate. Tumor-immune cell interactions on the other hand, are modeled at the cellular level: since we are interested here in explaining tumor morphologies and specific immune cell recruitment, the explicit modeling of the movements and interactions of individual cells is important. Therefore, rules of evolution based on the state and location of individual cells are derived and implemented, (see section 2).

A number of previous mathematical models have also coupled tumor growth with immune system dynamics. The majority of the coupled models are fully deterministic and are comprised of a series of ODEs or PDEs describing the dynamics of, for example, tumor cells, host cells and immune cells [6,19,21,28,37,39,40,44,46-48,62]. Bellouquid and Delitala [9] model the dynamics of general populations of interacting cells using kinetic theory and non-equilibrium statistical mechanics before offering specific examples. Coupled tumor-immune system models, both cellular and sub-cellular level depictions, are reviewed by Adam and Bellomo [1] and discussed again later in [55]. In more recent works, immunotherapy has also been investigated using similar ODE models coupled with optimal control theory $[10,20,22]$.

Most of these tumor-immune system models are fully deterministic, and while some models consider spatial tumor-immune interactions, these often focus on very simple spatial domains $[44,46,48]$. Here we also model temporal evolutions but extend the spatial consideration to a two-dimensional domain employing a hybrid CA-partial differential equation modeling approach.

This deterministic-stochastic CA modeling approach has been successfully used in the past to model tumor growth, chemotherapeutic treatment and the effects of vascularization on tumor growth $[2,26,27,49]$. Here we use reaction-diffusion PDEs to describe chemical species such as growth nutrients, and a CA strategy to track the tumor cells as well as immune cell species. Together, these elements simulate the growth of the tumor and the interactions of the immune cells with the tumor-growth. 
The flexibility of this CA modeling strategy and its computational implementation are illustrated (and essential) here where we consider a number of different possible causes for tumor morphology changes.

Similar discrete models, for example the PDE inspired models of Anderson and Chaplain [4] and off-lattice approach of Drasdo and Loeffler [25], have been used in the study of tumor growth and related processes. The model in this paper uses phenomenologically sourced, probabilistic CA rules rather than behaviors taken directly from PDE approximations and is more structured spatially than the off lattice models.

Using the hybrid CA-PDE model, we consider four mechanisms:

i) nutrient consumption variations,

ii) cell-cell adhesion,

iii) tumor "gluttony" with regard to nutrients, and

iv) immune cell interactions

leading to different morphological structures for two-dimensional tumors. This is accomplished through the development of a model with cellular behaviors related to those described in the experimental literature and by considering the ODE models of tumorimmune system interactions developed in other works (such as [20,22,39]).

In section 2, we present the development of the mathematical model used in this study including a description of the CA rules. In section 3, representative simulations are presented in order to gauge the effects of the various mechanisms on the growth of tumors before a discussion and interpretation of the results is presented in section 4 .

\section{Model overview}

The model we implement is an extension of the CA hybrid model of Mallet and de Pillis [43]. The cell types and chemical species modeled are equivalent, with the exception that the current model additionally tracks necrotic tumor cells. This work also includes several new CA rules, other modified or enhanced rules and a number of new assumptions to allow for the modeling of the different mechanisms responsible for tumor morphology changes.

The nutrient species and cell populations we track with this model are outlined in table 1. As in [59], we assume the presence of two nutrient types, one necessary for mitosis, and one necessary for survival. The sections that follow outline the model implemented in this research.

Table 1. Nutrients and cell types in model.

\begin{tabular}{ll}
\hline Variable & \multicolumn{1}{c}{ Description } \\
\hline$N(x, y, t)$ & Mitosis nutrient concentration \\
$M(x, y, t)$ & Survival nutrient concentration \\
$H(x, y, t)$ & Normal host cell number \\
$T(x, y, t)$ & Live tumor cell number \\
$T_{\text {nec }}(x, y, t)$ & Necrotic tumor cell number \\
$\mathrm{NK}(x, y, t)$ & Total NK immune cell number \\
$L(x, y, t)$ & Total CTL immune cell number \\
\hline
\end{tabular}




\subsection{Nutrient diffusion}

The following assumptions have been made in order to model the evolution of nutrients over time and in space.

- Both nutrient species are governed by reaction-diffusion equations of the same form, and diffusion rates for the two nutrients are equal.

- Normal tissue and immune cells consume both nutrients at the same rate $\alpha^{2}$ $\left(\right.$ time $^{-1}$ cell $^{-1}$ ).

- The consumption rate of both nutrients by tumor cells is a constant multiple of the normal and immune cell consumption rates. These factors are denoted by $\lambda_{M}$ and $\lambda_{N}$.

- Nutrients diffuse from a blood vessel on the boundary of the computational domain. Nutrient concentrations at the outer blood vessel wall are assumed to be constant.

The diffusion of the nutrients through tissue is modeled by the following nondimensionalized reaction-diffusion equations, as given in [43]:

$$
\begin{gathered}
\frac{\partial N}{\partial t}=\nabla^{2} N-\alpha^{2}(H+\mathrm{NK}+L) N-\lambda_{N} \alpha^{2} T N \\
\frac{\partial M}{\partial t}=\nabla^{2} M-\alpha^{2}(H+\mathrm{NK}+L) M-\lambda_{M} \alpha^{2} T M .
\end{gathered}
$$

The derivation of the non-dimensionalized equations and the justifications for the parameter choices and simplifications can be found in [43].

\subsection{Cell dynamics}

As mentioned in the introduction, while we model the diffusion of the nutrients with a system of reaction-diffusion equations, we model the action of the cell populations using local rules that are both probabilistic and deterministic. Cells will die, proliferate, or move depending on both the local concentration of nutrients near the cell and the states of neighboring cell populations. The parameters implemented for this portion of the model are outlined in table 2 .

2.2.1 Tumor cells. In this model, tumor cells are allowed to divide, move, or die, depending upon nutrient levels, the presence of immune cells, and forces due to cell-cell adhesion. Tumor cells die in one of two ways: either they are killed by cytotoxic lymphocytes, or they die due to insufficient nutrient; in this model, as in [43], cells do not undergo apoptosis. Each of these changes of state is governed by a probabilistic rule, which can be a function of nutrient concentrations, $M$ and $N$, as well as immune cell populations, NK and $L$.

Of the six probability functions summarized in this section, $P_{L}, P_{\mathrm{LD}}$ and $P_{\mathrm{NK}}$ have the same forms and functions as those in [43]. However, $P_{\text {del }}, P_{\text {div }}$ and $P_{\text {imdth }}$ play the same roles but have different forms. For completeness, therefore, we provide the formulas for all of the probability functions. To give an idea of the shape of each probability function, sample plots are provided in figure 1.

Tumor cell death due to insufficient nutrient. Tumor cell populations are updated at each proliferation time-step by considering each grid element containing a tumor cell in turn, 
Table 2. Description of model parameters used in the hybrid model. Entries marked with a (*) are new additions to the model, or significantly modified, while all other entries are also components of the model presented in [43].

\begin{tabular}{|c|c|}
\hline Parameter & Description \\
\hline$\lambda_{N}$ & $\begin{array}{l}\text { Multiplier for cancer cell consumption of mitosis nutrient } N \text { (cancer consumption rate of } \\
\quad N=\lambda_{N} \times \text { normal cell consumption rate of } N \text { ) }\end{array}$ \\
\hline$\lambda_{M}$ & $\begin{array}{l}\text { Multiplier for cancer cell consumption of survival nutrient } M \text { (cancer consumption rate of } \\
M=\lambda_{M} \times \text { normal cell consumption rate of } M \text { ) }\end{array}$ \\
\hline$\theta_{\text {div }}$ & $\begin{array}{l}\text { Parameter inversely related to probability of tumor cell division: increasing } \theta_{\text {div }} \text { decreases } \\
\text { chance of division }\end{array}$ \\
\hline$N_{\min }(*)$ & $\begin{array}{l}\text { Tumor cell division threshold: if nutrient concentration is less than } N_{\min } \text {, probability } \\
\text { of tumor cell division is } 0\end{array}$ \\
\hline$\theta_{\text {del }}$ & $\begin{array}{l}\text { Parameter affecting probability of cell death: increasing } \theta_{\text {del }} \text { increases chance of tumor } \\
\text { cell death }\end{array}$ \\
\hline$M_{\min }(*)$ & $\begin{array}{l}\text { Tumor cell death threshold: if nutrient concentration is less than } M_{\min } \text {, probability of } \\
\text { tumor cell death is } 1\end{array}$ \\
\hline$I_{0}$ & Background ratio of natural killer cells: (Initial \# NK cells) /(Total \# Grid Elements) \\
\hline$\theta_{L}$ & $\begin{array}{l}\text { Parameter inversely related to CTL induction: increasing } \theta_{L} \text { decreases chance } \\
\text { of induction of CTLs by tumor cells }\end{array}$ \\
\hline$\theta_{\mathrm{LD}}$ & Parameter for CTL death probability curve: increasing $\theta_{\mathrm{LD}}$ increases chance of CTL death \\
\hline$\theta_{N}(*)$ & $\begin{array}{l}\text { Probability of CTL induction by NK cells: increasing } \theta_{N} \text { increases chance of } \\
\text { induction of CTLs signaled by NK-tumor interactions }\end{array}$ \\
\hline$C_{\text {opt }}(*)$ & The optimal (energy minimizing) number of tumor cells in a grid site \\
\hline$I_{\mathrm{s}}(*)$ & $\begin{array}{l}\text { Overall immune strength against tumor cells: the smaller the parameter, the higher } \\
\text { the number of immune cells required in the neighborhood of a tumor } \\
\text { cell to kill that cell }\end{array}$ \\
\hline CTLkill & Number of times one CTL can attack tumor cells before deactivation \\
\hline NKkill & Number of times one NK cell can attack a tumor cell before deactivation \\
\hline
\end{tabular}

using a random ordering of elements at each cell-cycle. Within each grid element, all tumor cells can potentially change states. First, each tumor cell dies with probability $P_{\text {del }}$ (see figure 1), a function of the local concentration of the "survival" nutrient, $M$ :

$$
P_{\text {del }}=\exp \left[-\mathcal{H}\left(M-M_{\text {min }}\right)\left(\frac{M-M_{\text {min }}}{\theta_{\text {del }}}\right)^{2}\right],
$$

where $\mathcal{H}$ represents the Heaviside step function:

$$
\mathcal{H}(x)= \begin{cases}0 & \text { if } x<0 \\ 1 & \text { if } x \geq 0\end{cases}
$$

The use of the Heaviside function in $P_{\text {del }}$ forces death of the tumor cell if nutrient concentration $M$ is below the death threshold, $M_{\min }$. Dead cells become part of the necrotic population, and are removed from the living tumor population.

Tumor cell division due to sufficient nutrient. Next, those tumor cells that do not undergo necrosis are eligible to divide: each living tumor cell produces a daughter cell with probability $P_{\text {div }}$ (figure 1), a function of the local concentration of the "mitosis" nutrient, $N$ :

$$
P_{\text {div }}=1-\exp \left[-\mathcal{H}\left(N-N_{\text {min }}\right)\left(\frac{N-N_{\text {min }}}{\theta_{\text {div }}}\right)^{2}\right] \text {, }
$$



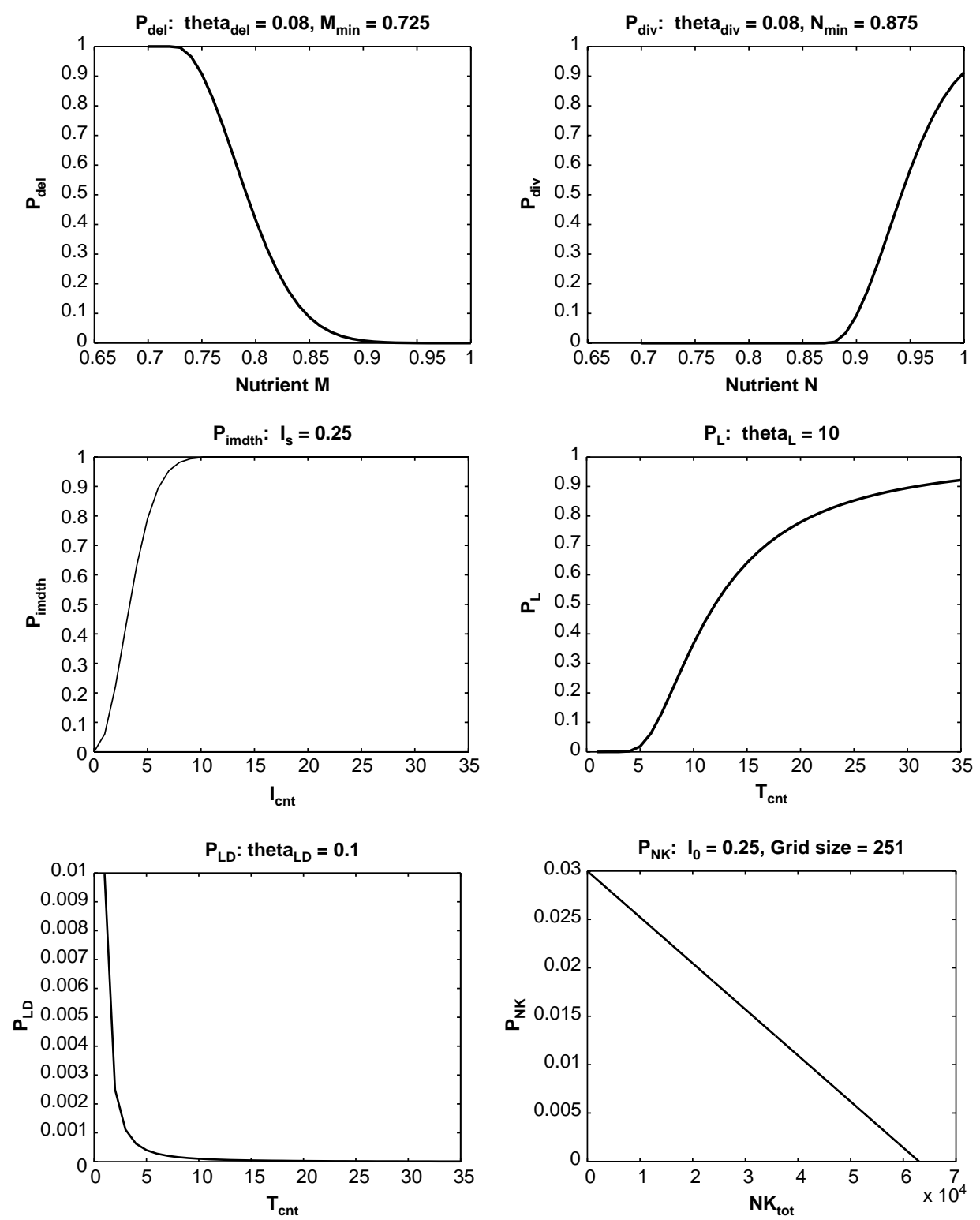

Figure 1. Plots of probability curves: Top: $P_{\text {del }} P_{\text {div }}$ Center: $P_{\text {imdth }}, P_{L}$. Bottom: $P_{\mathrm{LD}}, P_{\mathrm{NK}}$.

where $\mathcal{H}$ is the Heaviside step function (see equation (4)). Cell division is only possible if the concentration of nutrient is above the threshold $N_{\min }$, after which the higher the concentration of nutrient $N$, the higher the likelihood of cell division.

Tumor cell death due to immune cells. After cell division, living tumor cells are distributed among the elements in a $3 \times 3$ neighborhood of the grid element according to an energy minimizing adhesion rule, described below. As in [43], $P_{\text {imdth }}$, the probability that a tumor cell will be lysed by either the NK cells or the CTLs, is given by 


$$
P_{\text {imdth }}=1-\exp \left[-\left(I_{\mathrm{cnt}} I_{\mathrm{s}}\right)^{2}\right] .
$$

Here $I_{\text {cnt }}$ is the total number of immune cells, both NK cells and CTLs, in the neighborhood of the relevant tumor cell: $I_{\mathrm{cnt}}=\sum_{\mathrm{nbhd}}(\mathrm{NK}+L)$. We consider this local neighborhood to include the eight grid elements surrounding the grid site of interest, but further experimental information could result in modifications of the definition of this neighborhood.

The rules for immune cell kill of tumor cells are further described in section 2, in which the evolution of the immune cell populations is discussed.

Modeling cell-cell adhesion and invasion. This model attempts to capture the behavior of proliferating tumor cells as they become crowded and invade neighboring grid elements. It is important to consider the effects of cellular adhesion between cells and the extra-cellular matrix (ECM) since the secretion or suppression of adhesion molecules is a possible mechanism for the onset of metastasis [3,29,33]. Mathematical models incorporating adhesion include $[12,42,56]$, while $[17,60]$ explore the relationship between cell-cell adhesion, tumor morphology, hypoxia and disease prognosis.

Turner [63,64], provides a method for estimating the diffusion coefficient for biological cells modeled as adhesive, deformable spheres by considering the "potential energy of interaction" between individual cells. Turner shows that the diffusion coefficient is proportional to the second derivative of the grid element's potential energy function, $E(T)$,which can be derived in terms of the biological parameters of individual cells, such as elasticity and adhesiveness, (see figure 2). In this model we adapt Turner's method to the CA framework by redistributing living tumor cells after each proliferation step according to the following rule. The energy function is parametrized by its minimizing value, denoted by

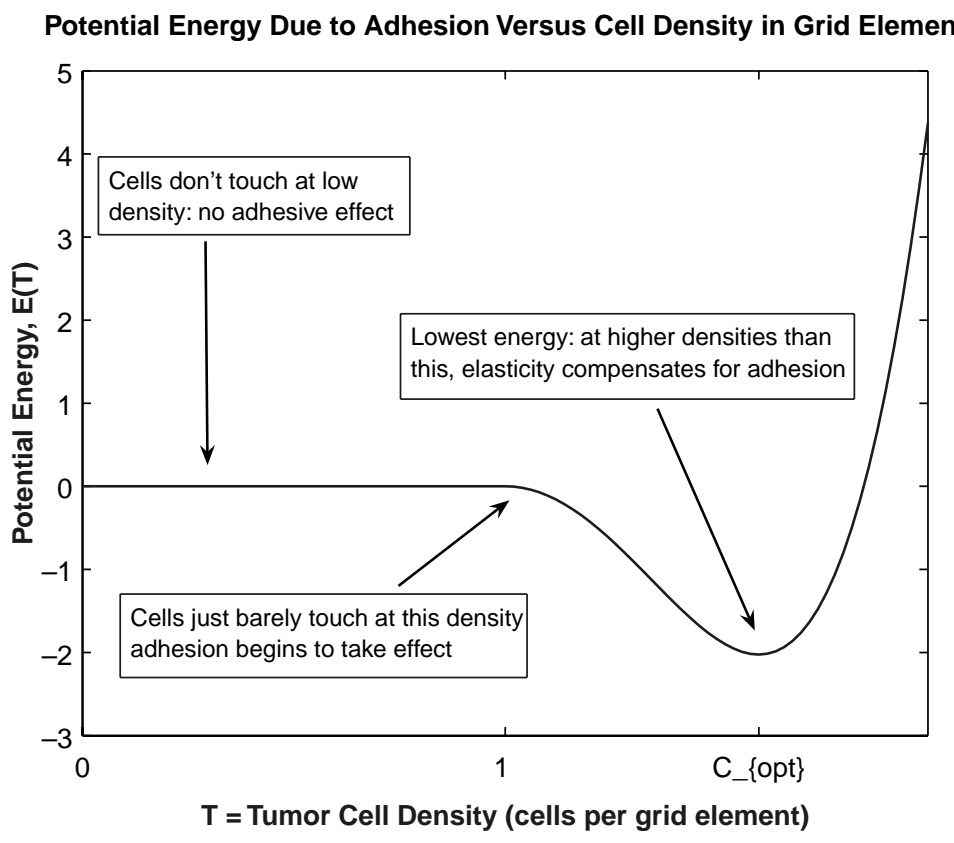

Figure 2. Potential energy function, $E(T)$. 
$C_{\text {opt }}$. If a newly updated grid element contains more than $C_{\text {opt }}$ cells, excess cells are distributed in the element's $3 \times 3$ neighborhood by moving cells to any element with fewer than $C_{\mathrm{opt}}$ cells, in order of decreasing cell density. This way, elements with cell populations closest to $C_{\text {opt }}$ are filled up to $C_{\text {opt }}$ first. If, after this initial redistribution, the center grid element still contains more than $C_{\mathrm{opt}}$ cells, living cells are evenly distributed, one by one, in a random order, to neighboring grid elements. The center grid element is not allowed to contain more than $C_{\text {opt }}$ cells. This provides a mechanism for inducing metastasis; for example, by setting $C_{\mathrm{opt}}$ to zero, living tumor cells are forced to metastasize.

2.2.2 Immune cells. NK cells are part of the innate immune response, and in this model are present at all times. They are allowed to roam freely and randomly through the computational grid, taking action when they do encounter a tumor cell. As in [43], in order to maintain the background immune system level, we impose a rule to generate new immune cells.

NK cell production. The homeostatic level of natural killer (NK) cells is determined by a fixed parameter of the system, denoted by

$$
I_{0}=\frac{\text { Initial \# NK cells }}{\text { Total \# Grid elements }} .
$$

At each cell-cycle, new NK cells are generated in any grid element without an NK cell in it with probability $P_{\mathrm{NK}}$ (see figure 1).

The probability that a new NK cell will be produced is given by

$$
P_{\mathrm{NK}}=I_{0}-\frac{\text { Current \# NK Cells }}{\text { Total \# Grid Elements }} .
$$

$P_{\mathrm{NK}}$ is the difference of the $a$ priori specified background ratio of NK cells to normal cells and the current NK to normal cell ratio. The likelihood of generating a new NK cell goes down as the total NK cell count goes up.

CTL induction. Unlike NK cells, CTLs are specific killers, and so they must be primed and recruited to the region of the tumor through various signals. To reduce computational requirements, we assume that NK cells and CTL cells do not occupy the same grid site at the same time. This then implies that a tumor cell is either killed by NK cells or by CTLs, but not by both simultaneously. This simplifying assumption is not a major hindrance to the functioning of the overall immune response to the tumor, but it is one that we wish to modify in future versions of this model.

The rules for the induction of CTLs in this model are as follows: if at least one tumor cell and one NK cell share the same grid site, then, with probability $\theta_{N}$ we induce the recruitment of CTLs to that location. Thus, the larger $\theta_{N}$, the greater the chance of recruiting CTLs to a location that presumably contains tumor cell detritus.

Similarly, if a tumor cell and CTLs share a location, more CTLs will be recruited to that spot, with a probability, $P_{L}$ (see figure 1). This probability depends on the local tumor density, and is given by

$$
P_{L}= \begin{cases}\exp \left[-\left(\frac{\theta_{L}}{T_{\mathrm{cnt}}}\right)^{2}\right], & T_{\mathrm{cnt}}>0 \\ 0, & \text { otherwise }\end{cases}
$$


Here, $T_{\mathrm{cnt}}$ is the number of tumor cells in the $3 \times 3$ square centered at the relevant grid element, and $\theta_{L}$ determines the shape of the probability curve.

Additionally, CTLs can be recruited to the immediate neighborhood surrounding the shared tumor-CTL grid site. The surrounding grid elements are sampled for further CTL induction. For each neighboring element that does not currently contain a CTL, a new CTL cell is created with probability $P_{L}$. This specific lymphocyte recruitment facilitates the satellitosis where $\mathrm{T}$ cells have been observed surrounding tumor cells undergoing apoptosis [61]. For completeness we note that for a neighboring tumor cell count of zero, $P_{L}$ is defined to be zero also.

In both cases, we note that CTL recruitment is possible as a result of either NK cells or CTL cells coming into contact with tumor cells, and does not require tumor cell lysis, as do the rules in [43].

When a tumor cell dies, in addition to updating the living and necrotic tumor populations, we distinguish between whether the tumor cell was killed by CTLs or NKs, and deactivate the immune cell, that is, reduce the immune cell count in that grid site accordingly.

If there is no cancer cell at a particular grid site, but there are immune cells, then the immune cells may move. Unlike in [43], we allow NK cells to move about "semi-blindly", that is, they move preferentially toward an immune-cell-free grid location, regardless of whether there is a tumor cell in that location or not. On the other hand, CTL cells move preferentially toward a tumor-occupied location.

CTL and NK cell death. As in [43], CTLs are assumed to die by one of two means. Firstly, they become functionally useless (and therefore, not tracked by the program) when their cell kill value CTLkill is reduced to zero. (As in [43], it is assumed that the cells of the specific immune system are able to lyse tumor cells more than once [38].) Secondly, the CTLs will die off or-equivalently as far as the implementation is concerned-move away from the domain of interest if they no longer detect tumor cells in their neighborhood. This cell removal occurs with probability $P_{\mathrm{LD}}$ (see figure 1):

$$
P_{\mathrm{LD}}= \begin{cases}1-\exp \left[-\left(\frac{\theta_{\mathrm{LD}}}{T_{\mathrm{cnt}}}\right)^{2}\right], & T_{\mathrm{cnt}}>0 \\ 0, & \text { otherwise }\end{cases}
$$

Here $\theta_{\mathrm{LD}}$ is a parameter determining the steepness of the probability curve. The probability of immune cell death is one when there are no tumor cells nearby.

NK cells, on the other hand, are removed in only one way: deactivation through NKtumor interactions. Currently, this occurs after an NK cell kills one tumor cell, since it is assumed that, unlike CTLs, each NK cell has the ability to attack only one tumor cell and then it is deactivated.

\section{Simulations and results}

In this section, we present simulations of various realizations of the model for different parameter sets, in order to test the effect on morphology of changing consumption rates, tumor gluttony, cell-cell adhesion and immune response. Two simulations with the same parameters will give different outcomes because of the probabilistic nature of the model. The simulations presented here are representative outcomes of typical simulation runs. 

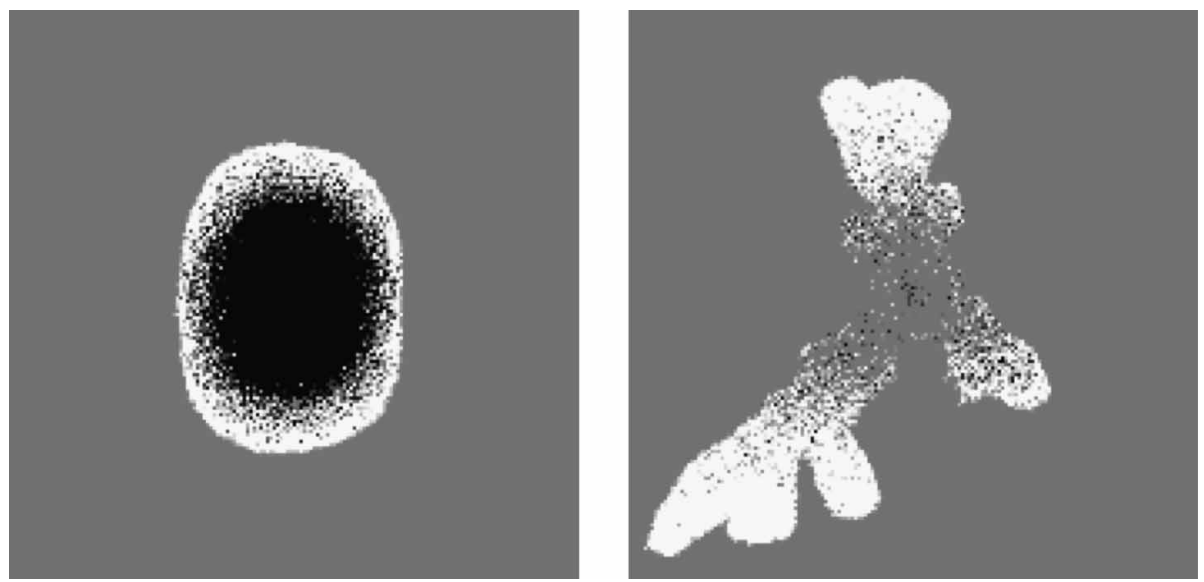

Figure 3. Tumor growth after 450 iterations with white tumor cells and black necrotic cells, for nutrient consumption rates $\lambda_{N}: 1.5$ (left) and 45 (right). $\lambda_{M}=1.5, N_{\min }=0.83, C_{\mathrm{opt}}=2, \theta_{\mathrm{div}}=\theta_{\mathrm{del}}=0.08, \theta_{L}=7, \theta_{N}=1$, $\theta_{\mathrm{LD}}=0.14, I_{0}=0.003, I_{\mathrm{s}}=0.25$, CTLkill $=1$, NKkill $=1, M_{\min }=0.725$.

\subsection{Effects of nutrient consumption rates}

Different rates of consumption of the mitotic nutrient have been considered previously by Mallet and de Pillis [43] and by Ferreira et al. [26]. Increasing the uptake of the nutrient by the tumor cells effectively increases their motility, since nutrient levels quickly become too low to support cell life at sites with high tumor density. Figure 3 shows characteristic growth patterns for different levels of nutrient consumption. On the left, the tumor consumes mitotic nutrient at a slower rate and hence can grow in density without moving far from the initial site of mutation. Note that this leads to the formation of a necrotic core in the interior of the tumor where nutrient levels have fallen. On the right, the tumor cells consume nutrient much faster than in the left hand simulation. To divide, the cells need to move away from the initial site of the tumor, toward the regions of higher nutrient concentration near the blood vessel, and a "fingered" growth develops. It is clear that varying the rate of tumor-cell nutrient consumption can lead to quite different tumor morphologies. It has been shown experimentally that a change in the tumor's microenvironment, in terms of $\mathrm{pH}$ or oxygenation, can affect a cell's ability to metabolize nutrient, [15]. Thus, manipulation of this micro-environment has been suggested as a treatment strategy, [30].

\subsection{Effects of tumor cell nutrient "Gluttony"}

Tumor morphology is also affected by the ratio between consumption of nutrients by tumor cells and the consumption by host and immune cells. For the two nutrients respectively, the ratio is provided immediately by the parameters $\lambda_{\mathrm{N}}$ and $\lambda_{\mathrm{M}}$. Figure 4 shows the spatial growth of a tumor with cells which consume both mitotic and survival nutrients at a much greater rate (100 times as fast) than do the normal host cells. The tumor evolves into a branched structure with cells actively searching for new areas with high nutrient levels, leaving behind them necrotic debris caused by tumor cell death due to a lack of nutrients. This is in contrast to the round-growth situation shown in the left side 


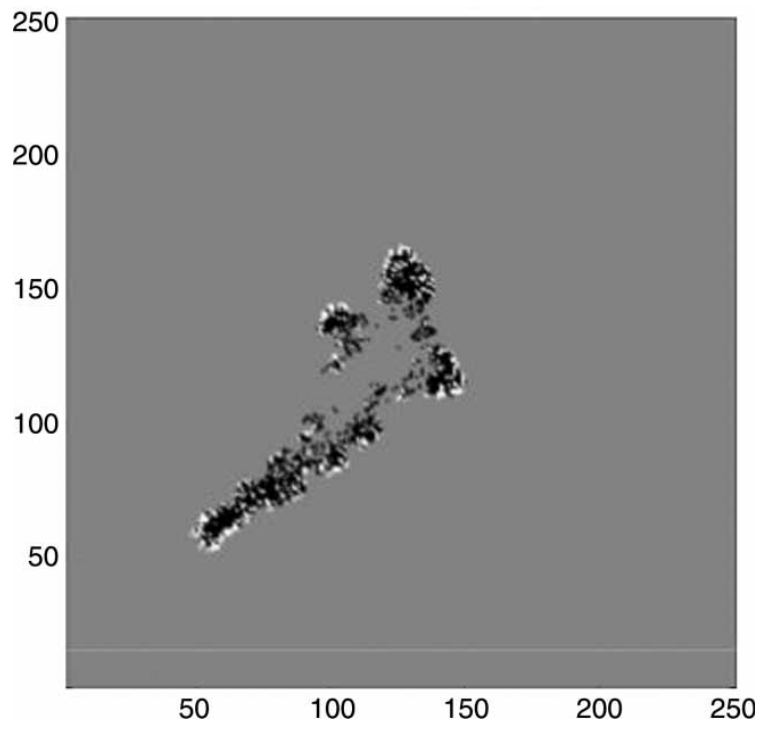

Figure 4. Tumor growth after 1000 iterations showing results of high tumor "gluttony" implemented through parameter values $\lambda_{N}=\lambda_{M}=100$. Other parameters are as given in figure 3 .

of figure 3 where tumor cells consume nutrients at nearly the same rate, (1.5 times as fast), as host cells.

Figure 5 shows that interestingly, a gluttonous approach to nutrient consumption is not always advantageous for the tumor. In fact, considering the first 20-30 iterations shown in the figure, the excessive consumption of nutrients could lead to quick tumor destruction. As the tumor tries to establish itself, the depletion of local nutrient levels reduces the possibility of cell division. Even if the tumor is able to establish high enough cell numbers to evade

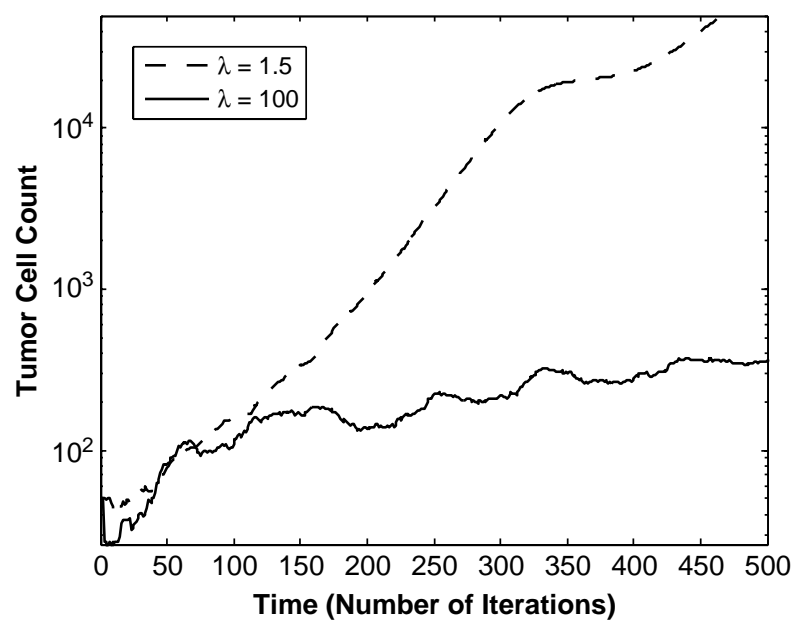

Figure 5. Number of tumor cells over time for two different gluttony levels- $\lambda_{M}=\lambda_{N}=1.5$ (default, dotted line) and $\lambda_{M}=\lambda_{N}=100$ (high gluttony, solid line). 


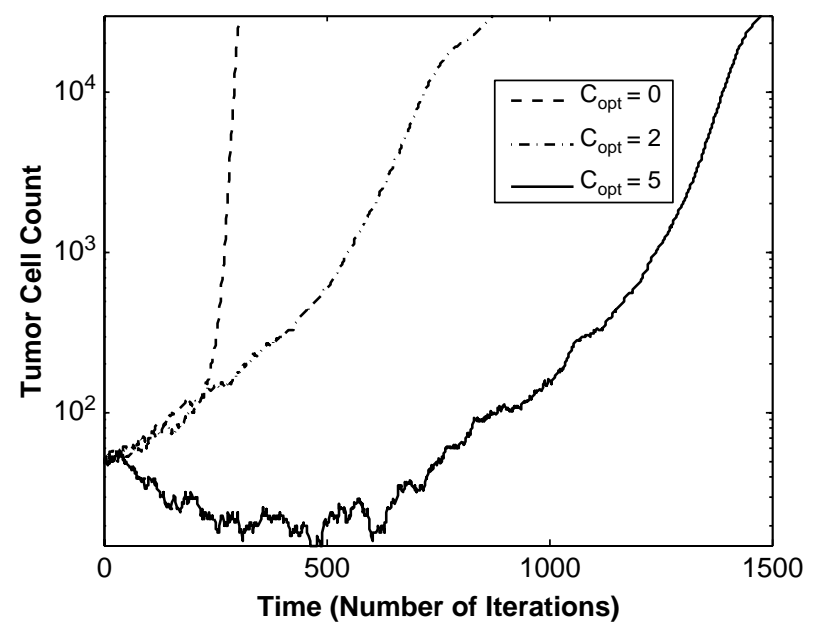

Figure 6. Tumor growth rate (number of tumor cells over time) as a function of the adhesion parameter, $C_{\mathrm{opt}}=0,2,5$. Other parameters are as given in figure 3 .

destruction completely, figure 4 shows that the cells are constantly in search of new nutrientrich areas of tissue which leads to the much lower, almost steady population of cells illustrated by figure 5 .

\subsection{Effects of weak versus strong adhesion}

Using the CA-adaptation of Turner's work, we also examined the effects of cell adhesion on the growth of tumors. Figure 6 shows the growth rates of tumors with three different values of the $C_{\text {opt }}$ parameter. This parameter determines when a location in the CA holds the optimum number of tumor cells and when the cells at that location would start spilling over into other locations. It is shown that the growth rate is significantly increased for lower values of $C_{\mathrm{opt}}$, and indeed for higher values of the parameter it appears quite possible that the tumor could be destroyed given other favorable circumstances, since the population nears extinction several times. The simulation suggests a possible mechanism for the initiation of metastasis via an alteration of cell adhesiveness. Experimental evidence for such a mechanism and its role in tumor growth is given in [35] and the references therein.

Figure 7 shows the spatial growth patterns of tumors with increasing tumor cell adhesion parameters. Lower values of $C_{\mathrm{opt}}$, such as the zero value discussed earlier, force tumor cells to move to other locations quickly, and take advantage of favorable nutrient conditions to divide and increase the overall population. For higher $C_{\text {opt }}$ values, the tumor cells adhere to each other, up to the optimal density, and are therefore more likely to remain in the location of the parent cell. However, the growth of the tumor population is hindered by the fact that the higher concentrations of cells consume more nutrients, and a necrotic core develops inside a (more or less) convex viable tumor rim.

\subsection{Effects of weak versus strong immune response}

Mallet and de Pillis [43] discussed the effects of the immune system on the growth rate of tumors and to a lesser extent the growth patterns. Here we see results regarding the effects of 

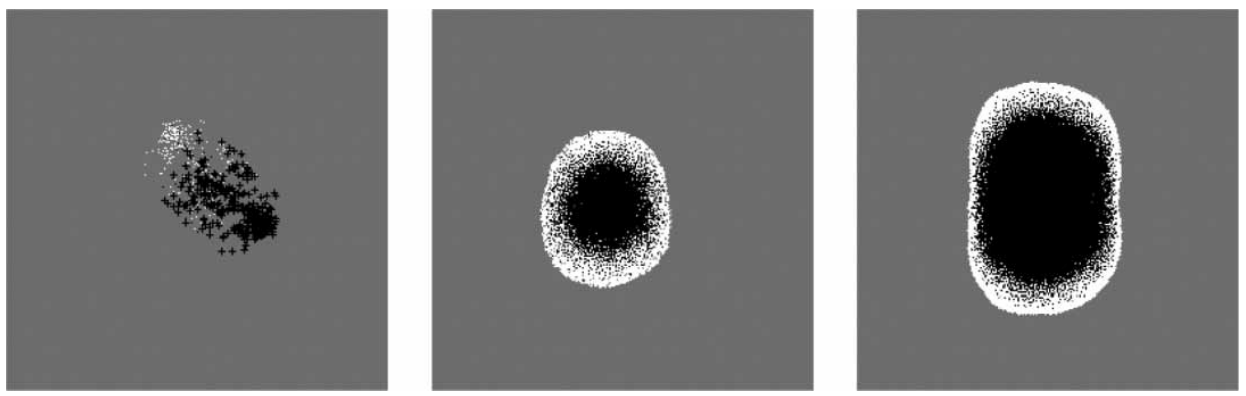

Figure 7. Tumor growth patterns for various levels of adhesion. Left: $C_{\mathrm{opt}}=0$ and 250 iterations; center: $C_{\mathrm{opt}}=2$ and 1500 iterations; right: $C_{\mathrm{opt}}=5$ and 1000 iterations. Other parameters are as given in figure 3 . Necrotic cells are shown in black and living cells are shown in white.

different background immune system levels and CTL induction strengths on tumor growth. In figure 8 , a stronger natural immune system $\left(I_{0}=0.003\right)$ is capable of controlling tumor growth and rebound. The simulation shown on the right of this figure shows that if the tumor can be detected by the immune system, it might decrease in size, but then can return to a second growth phase evading the immune system's control.

Figure 9 shows similar results to those found in [43] where a stronger recruitment of CTL cells $\left(\theta_{L}=3\right)$ induces a faster and more effective immune response to the tumor. The weaker response shown for $\theta_{L}=15$ initially controls the tumor but the immune system is soon overwhelmed and the tumor growth resumes. Note that while the CTL population does grow, it does not happen quickly enough to control the tumor growth.

Figure 10 is the spatial description at 40 and 80 iterations of the same simulation shown in figure 9. This shows the effect of the immune system, and in particular, the induction of CTL cells to the site of the growth, on the morphology of the tumor. The immune cells appear to surround then lyse the tumor cells for the case of strong induction. This is consistent with the observations of [18] and [34] who note that in spontaneous regression/complete resistance (SR/CR) mice, cancer cells induce infiltration of effector cells that form rosettes around the cancer cells, followed by cancer cell destruction. For the weaker immune cell induction case, some tumor cells are lysed, but most escape the immune attack, and continue to spread and divide as described in previous sections.
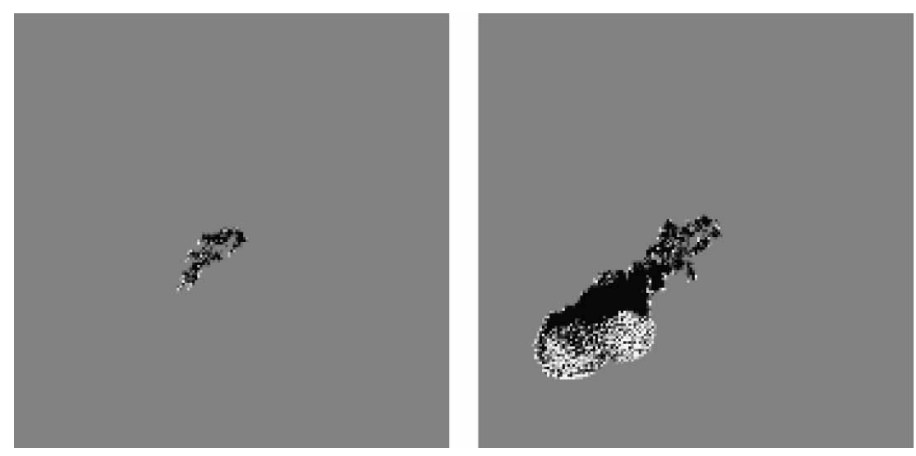

Figure 8. Tumor growth after 700 iterations for two different underlying immune strengths: $I_{0}=0.003$ (left) and $I_{0}=0.001$ (right), with $\theta_{L}=7$. Living cells are shown in white and necrotic cells in black. 

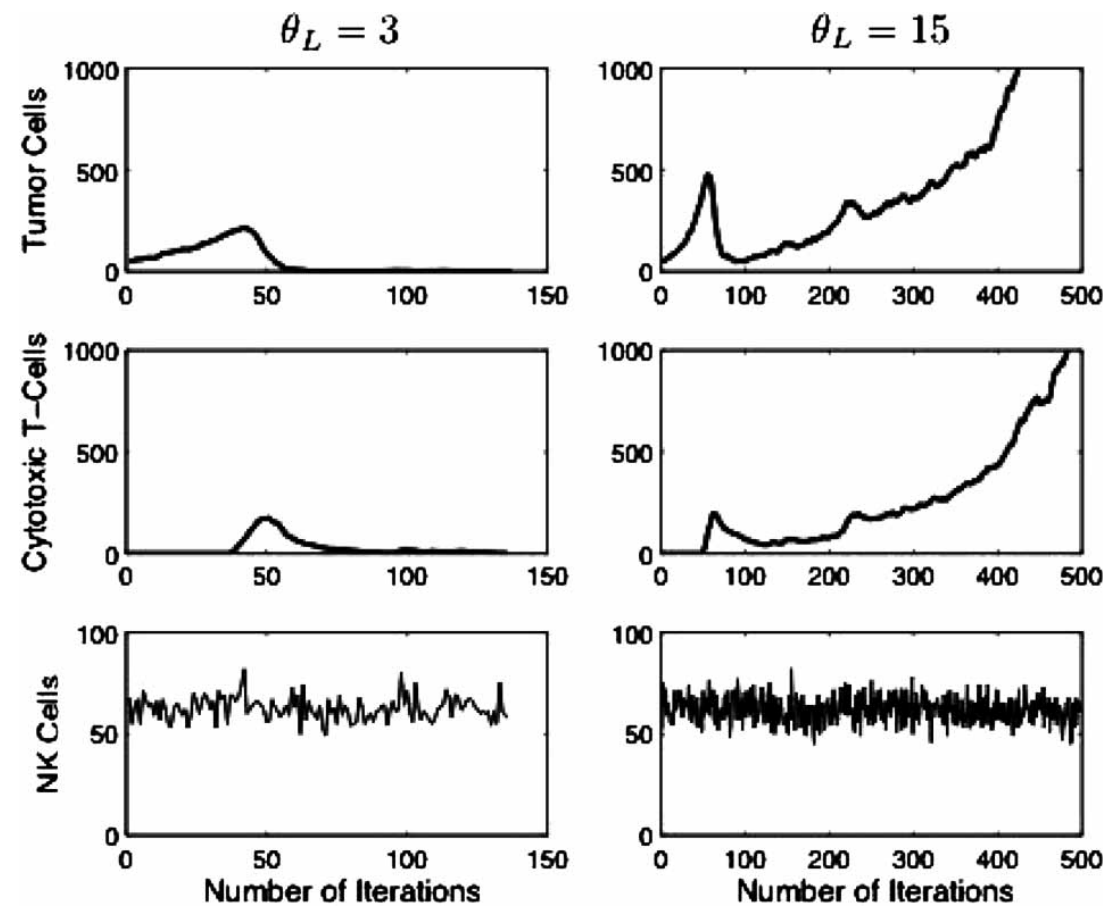

Figure 9. Comparison of the cell populations (tumor, CTL, NK) over time for two different values of the CTL induction parameter, $\theta_{L}=3$ and $15 . I_{0}=0.003$ (default) in both cases.
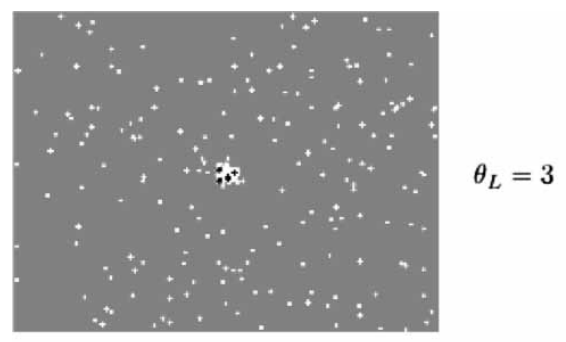

After 40 iterations

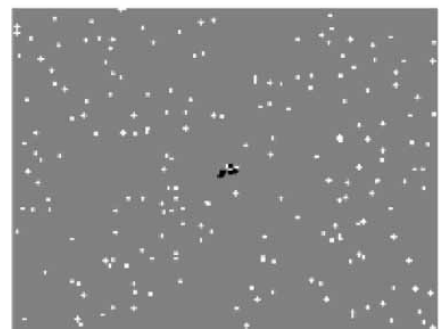

$\theta_{L}=15$

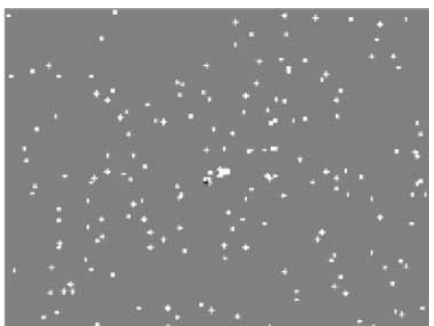

After 80 iterations

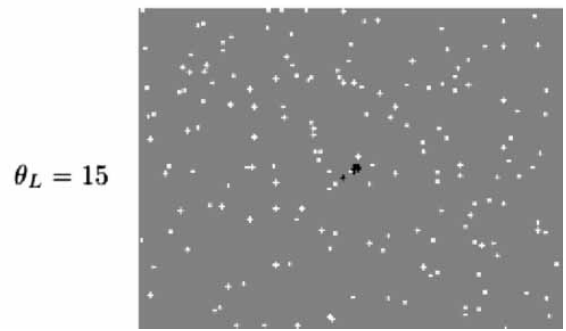

Figure 10. Spatial view of the simulations described in figure 9 at 40 and 80 iterations. Living tumor cells are shown in black and immune cells are shown in white. 


\section{Discussion}

The morphological development of a growing tumor can be significant when investigating factors such as staging, potential for metastasis, immune cell infiltration, and treatment effectiveness. The CA-hybrid model of tumor growth we have presented in this work provides a set of rules that allow us to investigate spatio-temporal tumor growth in the context of nutrient consumption rates, cell-cell adhesion and both innate and specific immune responses. We have shown that each of these factors has some effect on the morphology of the growing tumor, as well as on the tumor's ability to establish itself.

In future versions of this model, we plan to incorporate further rules to reflect more details of the biology at various scales. For example, we are currently working on the incorporation of rules that drive cell-cycle dynamics and tumor invasion of normal tissue as a function of realistic oxygen and glucose consumption rates, ATP production and waste excretion. Additionally, for investigation of chemotherapy and immunotherapy treatments, we are including vascular structures, and the effects of vascular growth and collapse.

Computational constraints are currently a major reason for running all the simulations presented in this paper in two dimensions, instead of three. The computation of the diffusion of the two chemical species consumes the majority of the simulation time. We tracked the diffusion of nutrients by solving the steady state diffusion equation, a reasonable approach since diffusion of the relevant chemicals takes place on a much shorter time scale than the cell-cycle. This is an approach taken by others as well [26,27]. The significant time-sink in the computational solution of the steady-state diffusion equations is the sparse matrix solver. We tested multiple sparse matrix solvers, and found that the most efficient of the easy to implement solvers was BiCGStab. We note, however, that all the sparse matrix solvers encounter problems with break-down, and when that happens, another solver must be employed, generally a direct solver. This approach is not the most computationally efficient, but was the one used in [43]. We, therefore, implemented an alternate approach for the simulations presented in this paper. With this alternate approach, diffusion is calculated using discrete local rules, and concentrations are updated more often when changes in cell populations occur. Numerical tests showed that this last approach was much faster, and produced comparable results. Details of the numerical procedure will appear in a forthcoming paper. We are currently considering the exploration of a multi-grid approach in hopes that this will allow the extension of the current model to three space dimensions.

\section{Acknowledgements}

The authors would like to thank K.E.O. Todd-Brown for her contributions to significant portions of the cellular-automata code and C. Du Bois for his help in streamlining the diffusion code and in investigating cell-cell adhesion. The authors also extend thanks to the anonymous reviewers for insightful feedback. LD acknowledges partial support from the W.M. Keck Foundation and from the National Science Foundation NSF-0414011.

\section{References}

[1] Adam, J.A. and Bellomo, N, 1997, A Survey of Models for Tumor-immune System Dynamics (Cambridge, MA: Birkhauser).

[2] Alarcon, T., Byrne, H.M. and Maini, P.K., 2003, A cellular automaton model for tumour growth in inhomogeneous environment, Journal of Theoretical Biology, 225, 257-274. 
[3] Alt-Holland, A., Zhang, W., Margulis, A. and Garlick, J.A., 2005, Microenvironmental control of premalignant disease: the role of intercellular adhesion in the progression of squamous cell carcinoma, Seminars in Cancer Biology, 15, 84-96.

[4] Anderson, A.R.A. and Chaplain, M.A.J., 1998, Continuous and discrete mathematical models of tumor induced angiogenesis, Bulletin of Mathematical Biology, 60, 877-900.

[5] Araujo, R.P. and McElwain, D.L.S., 2004, A history of the study of solid tumour growth: The contribution of mathematical modelling, Bulletin of Mathematical Biology, 66, 1039-1091.

[6] Arciero, J.C., Jackson, T.L. and Kirschner, D.E., 2004, A mathematical model of tumor-immune evasion and siRNA treatment, Discrete and continuous dynamical systems—series B, 4(1), 39-58.

[7] Ermentrout, Bard, G. and Edelstein-Keshet, L., 1993, Cellular automata approaches to biological modeling, Journal of Theoretical Biology, 160, 97-133.

[8] Bellomo, N. and Maini, P., 2005, Preface, Mathematical models and methods in applied sciences, 15(11), iii-viii.

[9] Bellouquid, A. and Delitala, M., 2005, Mathematical models and tools of kinetic theory towards modelling complex biological systems, Mathematical models and methods in applied sciences, 15(11), 1639-1666.

[10] Burden, T., Ernstberger, J., Fister, K. and Renee, 2004, Optimal control applied to immunotherapy, Discrete and continuous dynamical systems-series B, 4(1), 135-146.

[11] Burton, A.C., 1966, Rate of growth of solid tumours as a problem of diffusion, Growth, 30, 159-176.

[12] Byrne, H.M. and Chaplain, M.A.J., 1996, Modelling the role of cell-cell adhesion in the growth and development of carcinomas, Mathematical and Computer Modelling, 24(12), 1-17.

[13] Byrne, H.M. and Chaplain, M.A.J., 1997, Free boundary value problems associated with the growth and development of multicellular spheroids, European Journal of Applied Mathematics, 8, 639-658.

[14] Callewaert, D.M., Meyers, P., Hiernaux, J. and Radcliff, G., 1988, Kinetics of cellular cytotoxicity mediated by cloned cytotoxic T lymphocytes, Immunobiology, 178, 203-214.

[15] Casciari, J.J., Sotirchos, S.V. and Sutherland, R.M., 1992, Variations in tumor growth rates and metabolism with oxygen concentration, glucose concentration, and extra-cellular pH, Journal of Cell Physiology, 151, 386-394.

[16] Cristini, V., Lowengrub, J. and Nie, Q., 2003, Nonlinear simulation of tumor growth, Journal of Mathematical Biology, 36, 191-224.

[17] Cristini, V., Frieboes, H.B., Gatenby, R., Caserta, S., Ferrari, M. and Sinek, J., 2005, Morphological instability and cancer invasion, Clinical Cancer Research, 11, 6772-6779.

[18] Cui, Z., Willingham, M.C., Hicks, A.M., et al., 2003, Spontaneous regression of advanced cancer: Identification of a unique genetically determined, age-dependent trait in mice, PNAS, 100(11), 6682-6687.

[19] De Angelis, E. and Jabin, P.E., 2003, Qualitative analysis of a mean field model of tumor-immune system competition, Mathematical models and methods in applied sciences, 13(2), 187-206.

[20] de Pillis, L. and Radunskaya, A., 2001, A mathematical tumor model with immune resistance and drug therapy: An optimal control approach, Journal of Theoretical Medicine, 3, 79-100.

[21] de Pillis, L.G. and Radunskaya, A., 2003, A mathematical model of immune response to tumor invasion. Proceedings of the Second MIT Conference on Computational Fluid and Solid Mechanics. In: K.J. Bathe (Ed.) Computational Fluid and Solid Mechanics.

[22] de Pillis, L.G. and Radunskaya, A., 2003, The dynamics of an optimally controlled tumor model: A case study, Mathematical and Computer Modelling, 37, 1221-1244.

[23] Dormann, S. and Deutsch, A., 2002, Modeling of self-organized avascular tumor growth with a hybrid cellular automaton, In Silico Biology, 2, 0035.

[24] Dutta, A. and Popel, A.S., 1995, A theoretical analysis of intracellular oxygen diffusion, Journal of Theoretical Biology, 176, 433-445.

[25] Drasdo, D. and Loeffler, M., 2001, Individual-based models on growth and folding in one-layered tissues: Intestinal crypts and early development, Nonlinear Analysis, 47, $245 Đ 256$.

[26] Ferreira, Jr., S, C., Martins, M.L. and Vilela, M.J., 2002, Reaction-diffusion model for the growth of avascular tumor, Physical Review E, 65, 021907.

[27] Ferreira, Jr., S, C., Martins, M.L. and Vilela, M.J., 2003, Morphology transitions induced by chemotherapy in carcinomas in situ, Physical Review E, 67, 051914.

[28] Galach, M., 2003, Dynamics of the tumor-immune system competition-the effect of time delay, International Journal of Applied Mathematics and Computer Science, 13(3), 395-406.

[29] Gassmann, P., Haier, J. and Nicolson, G.L., n.d, Cell adhesion and invasion during secondary tumor formation. Cancer Growth and Progression vol. 3, (Amsterdam: Kluwer).

[30] Gatenby, R. and Gawlinski, E.T., 2003, The glycolytic phenotype in carcinogenesis and tumor invasion: Insights from mathematical models, Cancer Research, 63, 3847-3854.

[31] Greenspan, H.P., 1972, Models for the growth of a solid tumor by diffusion, Studies in Applied Mathematics, 51, 317-338.

[32] Greller, L., Tobin, F. and Poste, G., 1996, Tumor Heterogeneity and progression: conceptual foundation for modeling, Invasion and Metastasis, 16, 177-208. 
[33] Haier, J. and Nicolson, G.L., 2001, Role of tumor cell adhesion as an important factor in formation of distant metastases, Diseases Colon Rect., 44, 876-884.

[34] Hicks, A.M., Riedlinger, G., Willingham, M.C., et al. 2006, Transferable anticancer innate immunity in spontaneous regression/complete resistance mice, PNAS, 103(20), 7753-7758.

[35] Jain, R.K., 1999, Transport of molecules, particles, and cells in solid tumors, Annual Review of Biomedical Engineering, 01, 241-263.

[36] Kansal, A.R., Torquato, S., Harsh, G.R. IV, Chiocca, E.A. and Deisboeck, T.S., 2000, Simulated brain tumor growth dynamics using a three dimensional cellular automaton, Journal of Theoretical Biology, 203, 367-382.

[37] Kirschner, D. and Panetta, J.C., 1998, Modeling immunotherapy of the tumor-immune interaction, Journal of Mathematical Biology, 37, 235-252.

[38] Kuznetsov, V.A., 1997, Basic models of tumor-immune system interactions-Identification, analysis and predictions. In: J.A. Adam and N. Bellomo (Eds.) A Survey of Models for Tumor-immune System Dynamics (Cambridge, MA: Birkhauser).

[39] Kuznetsov, V. and Knott, G., 2001, Modelling tumor regrowth and immunotherapy, Mathematical and Computer Modelling, 33(12/13), 1275-1287.

[40] Lin, A., 2004, A model of tumor and lymphocyte interactions, Discrete and continuous dynamical systemsseries $B, \mathbf{4}(1), 241-266$.

[41] Mallet, D.G., 2004, Mathematical modeling of the role of haptotaxis in tumour growth and invasion. PhD Thesis. Queensland University of Technology, Brisbane, Australia.

[42] Mallet, D.G. and Pettet, G.J., 2006, A mathematical model of integrin-mediated haptotactic cell migration, Bulletin of Mathematical Biology, 68(2), 231-253.

[43] Mallet, D.G. and de Pillis, L.G., 2006, A cellular automata model of tumor-immune system interactions, Journal of Theoretical Biology, 239, 334-350.

[44] Matzavinos, A. and Chaplain, M.A.J., 2004, Mathematical modelling of the spatio-temporal response of cytotoxic T-lymphocytes to a solid tumour, Mathematical Medicine and Biology, 21, 134.

[45] Merrill, S.J., 1982, Foundations of the use of enzyme kinetic analogy in cell-mediated cytotoxicity, Mathematical Biosciences, 62, 219-236.

[46] Owen, M.R. and Sherratt, J.A., 1997, Pattern formation and spatiotemporal irregularity in a model for macrophage-tumour interactions, Journal of Theoretical Biology, 189(1), 63-80.

[47] Owen, M.R. and Sherratt, J.A., 1998, Modelling the macrophage invasion of tumours: effects on growth and composition, IMA Journal of Mathematics Applied in Medicine and Biology, 15, 165-185.

[48] Owen, M.R., Byrne, H.M. and Lewis, C.E., 2004, Mathematical modelling of the use of macrophages as vehicles for drug delivery to hypoxic tumour sites, Journal of Theoretical Biology, 226(4), 377-391.

[49] Patel, A.A., Gawlinski, E.T., Lemiueux, S.K. and Gatenby, R.A., 2001, A cellular automaton model of early tumor growth and invasion: the effects of native tissue vascularity and increased anaerobic tumor metabolism, Journal of Theoretical Biology, 213, 315-331.

[50] Peirce, S., Van Gieson, E.J. and Skalak, T.C., 2004, Multicellular simulation predicts microvascular patterning and in silico tissue assembly, The FASEB Journal, express article 10.1096/fj.03-0933fje 4.

[51] Perelson, A.S. and Mackean, C.A., 1984, Kinetics of cell-mediated cytotoxicity: stochastic and deterministic multistage models, Journal of Mathematical Biology, 170, 161-194.

[52] Perelson, A.S. and Weisbuch, G., 1997, Immunology for physicists, Reviews of Modern Physics, 69(4), $1219-1267$.

[53] Peter, J. and Semmler, W., 2004, Integrating kinetic models for simulating tumor growth in monte carlo simulation of ECT systems, IEEE Transactions on Nuclear Science, 51(5), 2628-2633.

[54] Pettet, G.J., Please, C.P., Tindall, M.J. and McElwain, D.L.S., 2001, The migration of cells in multicell tumor spheroids, Bulletin of Mathematical Biology, 63, 231-257.

[55] Preziosi, L., 2003, Cancer Modelling and Simulation (Boca Raton: CRC-Press, Chapman Hall).

[56] dos Reis, A.N., Mombach, J.C.M., Walter, M. and de Avila, L.F., 2003, The interplay between cell adhesion and environment rigidity in the morphology of tumors, Physica A-Statistical Mechanics and its Applications, 322, $546-554$.

[57] Ribba, B., Alarcón, T., Marron, K., Maini, P.K. and Agur, Z., 2004, The use of hybrid cellular automaton models for improving cancer therapy. In: P.M.A. Sloot, B. Chopard and A.G. Hoekstra (Eds.) ACRI, LNC 2205 , pp. 444-453.

[58] Scalerandi, M., Romano, A., Pescarmona, G.P., Delsanto, P.P. and Condat, C.A., 1999, Nutrient competition as a determinant for cancer growth, Physical Review E, 59(2), 22206-22217.

[59] Seiden, P.E. and Celada, F., 1992, A model for simulating cognate recognition and response in the immune system, Journal of Theoretical Biology, 158(3), 328-357.

[60] Smolle, J., 1998, Cellular automaton simulation of tumour growth-equivocal relationships between simulation parameters and morphologic pattern features, Analytical Cellular Pathology, 17(2), 71-82.

[61] Soiffer, R., Lynch, T., Mihm, M., et al., 1998, Vaccination with irradiated autologous melanoma cells engineered to secrete human granulocyte macrophage colony-stimulating factor generates potent antitumor immunity in patients with metastatic melanoma, Proceedings of the National Academy of Sciences of the United States of America, 95, 13141-13146. 
[62] Takayanagi, T. and Ohuchi, A., 2001, A mathematical analysis of the interactions between immunogenic tumor cells and cytotoxic T lymphocytes, Microbiology and Immunology, 45(1), 709-715.

[63] Turner, S., 2005, Using cell potential energy to model the dynamics of adhesive biological cells, Physical Review E, 71, 041903-1-041903-12.

[64] Turner, S., Sherratt, J.A., Painter, K.J. and Savill, N.J., 2004, From a discrete to a continuous model of biological cell movement, Physical Review E, 69(2), 1 Art. No. 021910 Part 1.

[65] Webb, S.D., Sherratt, J.A. and Fish, R.G., 1999, Mathematical modelling of tumour acidity: regulation of intracellular pH, Journal of Theoretical Biology, 196, 237-250. 


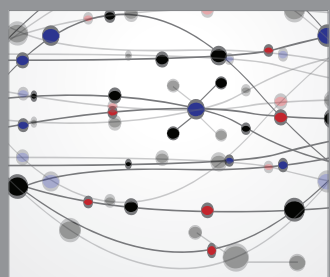

The Scientific World Journal
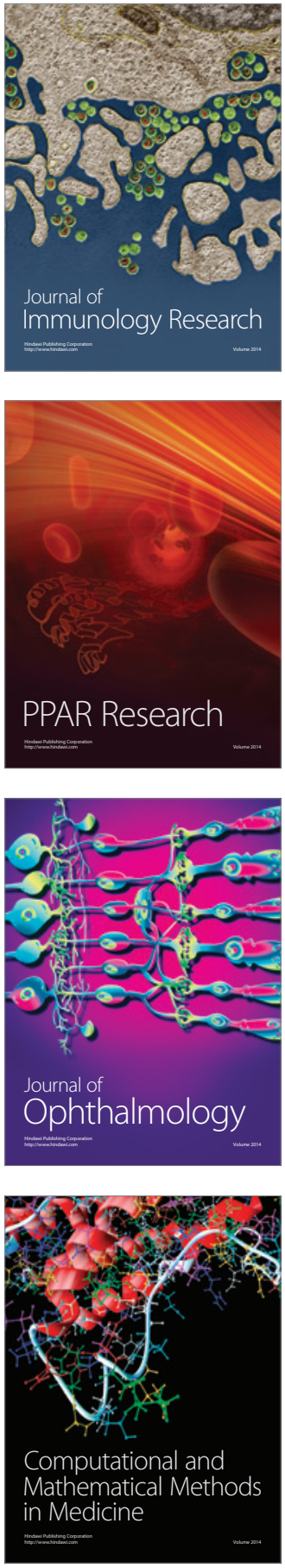

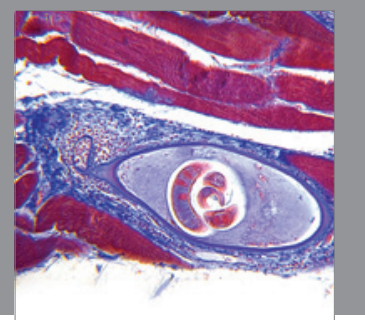

Gastroenterology

Research and Practice
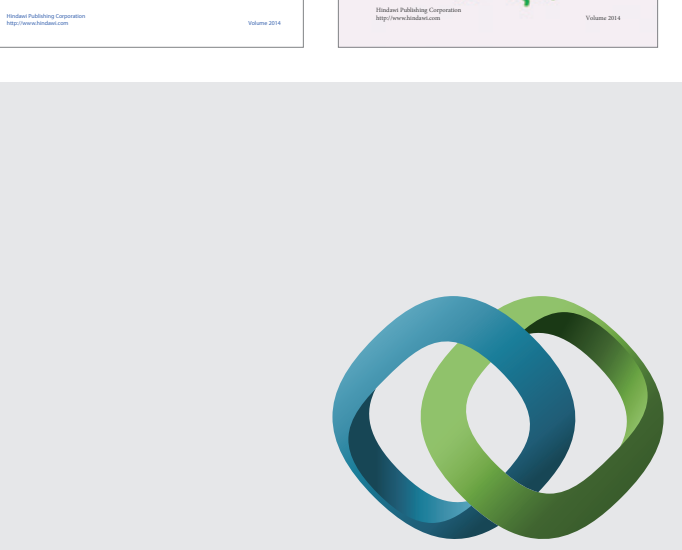

\section{Hindawi}

Submit your manuscripts at

http://www.hindawi.com
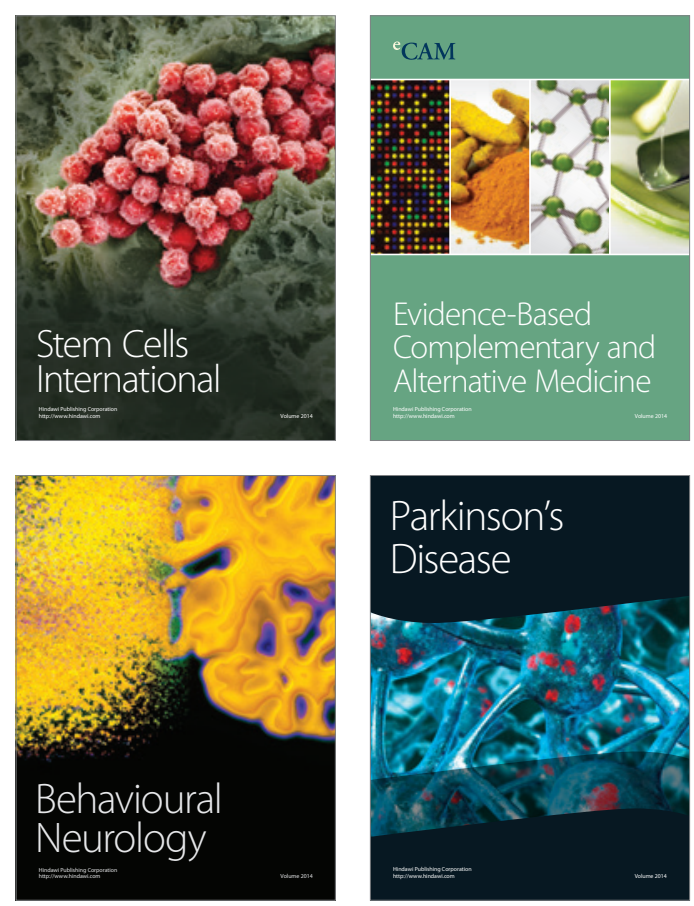

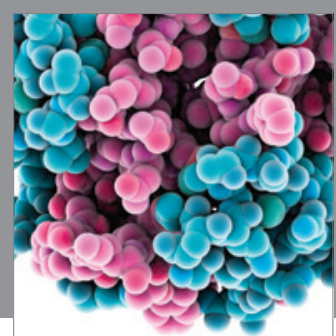

Journal of
Diabetes Research

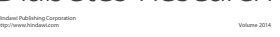



Disease Markers
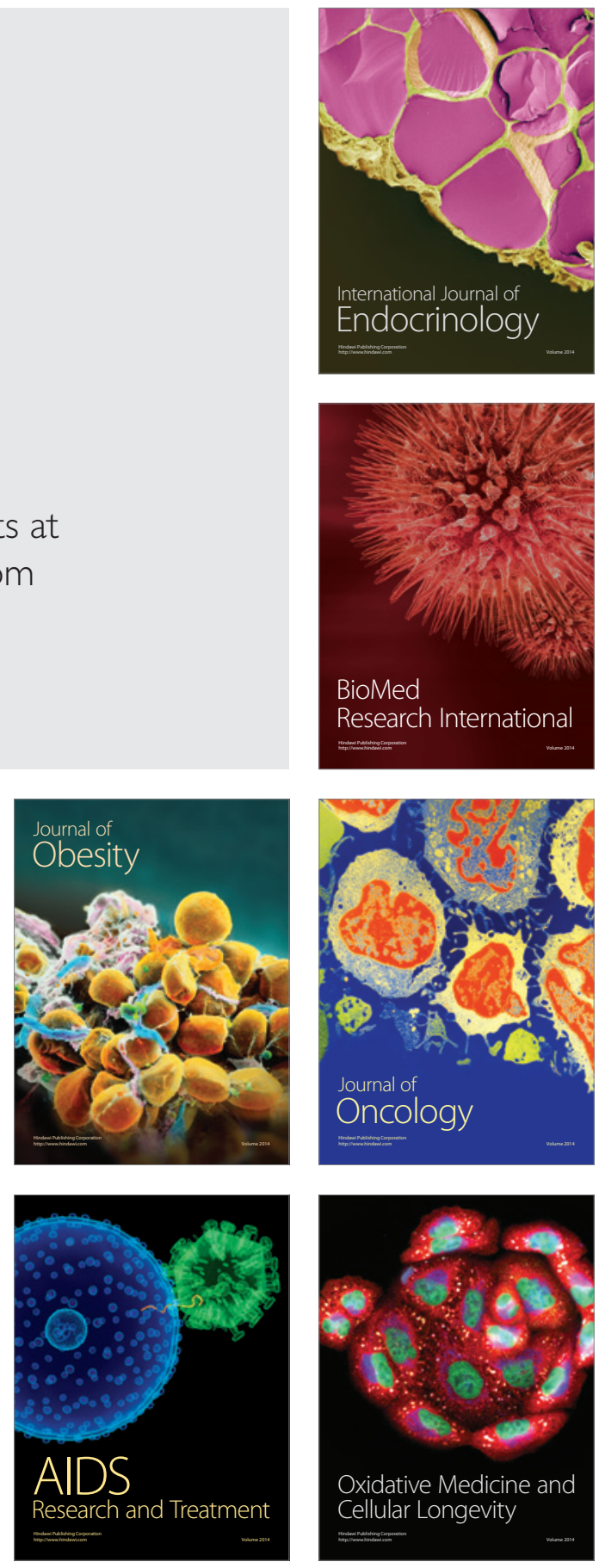